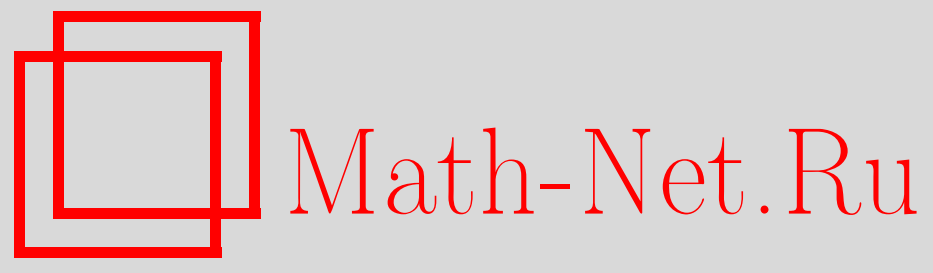

А. В. Зайцев, Ю. В. Соколкин, А. А. Фукалов, Решение задачи Ламе для составных трансверсальноизотропных сфер с общим центром, Вестн. Сам. гос. техн. ун-та. Сер. Физ.-мат. науки, 2021, номер 1, 83-96

DOI: https://doi.org/10.14498/vsgtu1830

Использование Общероссийского математического портала MathNet.Ru подразумевает, что вы прочитали и согласны с пользовательским соглашением

http://www.mathnet.ru/rus/agreement

Параметры загрузки:

IP : 18.234 .156 .22

26 апреля 2023 г., $11: 16: 16$

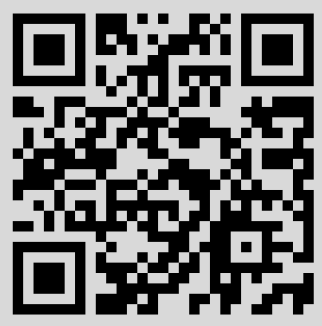


УДК 539.311

\title{
Решение задачи Ламе для составных трансверсально-изотропных сфер с общим центром
}

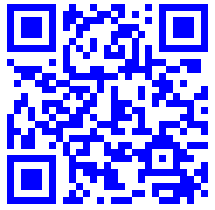

\author{
(C) А. В. Зайцев, Ю. В. Соколкин, А. А. Фукалов
}

Пермский национальный исследовательский политехнический университет, Россия, 614990, Пермь, Комсомольский пр-т, 29.

\section{Аннотация}

Получено точное аналитическое решение задачи Ламе о равновесии составного тела, состоящего из двух посаженных с натягом трансверсально-изотропных сфер с общим центром. Тело находится под действием равномерных внешнего и внутреннего давлений. Определено давление натяга на поверхности контакта в предположении, что оно является следствием различия в параметрах геометрии частей составной сферы. Проанализированы закономерности влияния анизотропии материалов (материальные постоянные удовлетворяют ограничениям в виде неравенств, обеспечивающих положительность собственных значений оператора упругости) и величины давления натяга на распределение напряжений в поперечных сечениях составных центрально-симметричных сосудов давления. Проведенная оценка влияния анизотропии материалов показала возможность «управления» величинами и характером распределения напряжений в составных конструкциях, оптимально соответствующих заданным режимам эксплуатации. Полученные результаты свидетельствуют, что изменение показателя анизотропии - увеличение его значений во внутренних или внешних частях сфер приводит к возрастанию или снижению абсолютных величин напряжений соответственно. Это увеличение или уменьшение показателей анизотропии материалов создаваемых конструкций может быть реализовано на этапе их проектирования благодаря изменению схемы армирования при сохранении свойств отдельных элементов структуры. На основе многокритериального подхода проведена оценка начальной прочности составных центрально-симметричных сосудов по механизмам растяжения или сжатия в радиальном и окружном направлениях. Установлено, что

\section{Научная статья}

ə () (i) Контент публикуется на условиях лицензии Creative Commons Attribution 4.0 International (https://creativecommons.org/licenses/by/4.0/deed.ru)

\section{Образец для цитирования}

З ай це в А. В., С ок ол ки н Ю. В., Ф ука л о в А. А. Решение задачи Ламе для составных трансверсально-изотропных сфер с общим центром // Вестн. Сам. гос. техн. ун-та. Сер. Физ.-мат. науки, 2021. Т. 25, № 1. С. 83-96. https://doi.org/10.14498/vsgtu1830.

\section{Сведения об авторах}

Алексей Вячеславович Зайцев (1) https://orcid.org/0000-0003-0578-7917

кандидат физико-математических наук, доцент; доцент каф. механики композиционных материалов и конструкций; e-mail: a-zaitsev@mail.ru

\section{Юрий Викторович Соколкин (1) https://orcid.org/0000-0003-3255-1360}

доктор физико-математических наук, профессор; профессор каф. механики композиционных материалов и конструкций

Антон Александрович Фукалов (10) https://orcid.org/0000-0003-3009-7379 старший преподаватель; каф. механики композиционных материалов и конструкций; e-mail:mr_aa@mail.ru 
увеличение давления натяга может привести к появлению областей материала, потерявших способность сопротивляться сжатию в окружном направлении. Эти области располагаются вблизи внутренней поверхности сосуда, на которой действует равномерно распределенное давление, меньшее по абсолютной величине по сравнению с внешним давлением. Обнаружено, что точки составного сосуда, находящиеся на поверхности контакта, становятся наиболее опасными с точки зрения возможности начала разрушения по механизму сжатия в радиальном направлении.

Ключевые слова: аналитическое решение, задача Ламе, составная трансверсально-изотропная сфера, анизотропия, многокритериальная оценка начальной прочности, составные сферические сосуды, контактное давление.

Получение: 5 октября 2020 г. / Исправление: 18 февраля 2021 г. Принятие: 10 марта 2021 г. / Публикация онлайн: 30 марта 2021 г.

1. Введение. Вопросы безопасной транспортировки и хранения жидких и газообразных сред не теряют своей актуальности на протяжении десятилетий. Одним из способов повышения эффективности, в т.ч. и экономической, используемых для этих целей сосудов является повышение рабочего давления. Это, в свою очередь, предопределяет повышенные требования к «живучести» и безопасной эксплуатации элементов конструкций, работающих при многократно изменяющихся внутренних давлениях в течение длительного времени. Увеличение толщины стенки конструкции, за счет чего оказывается возможным рост рабочего давления, не всегда оправдано. Это достигается применением многослойных композиционных материалов, обладающих естественной, ярко выраженной анизотропией свойств. Поэтому при проектировании современных сосудов давления используются решения, в которых конструкция и материал изготавливаются одновременно. Если конструкции будут еще и составными, то появится возможность управления величинами, характером и знаком напряжений за счет изменения схемы армирования. А это позволит создавать конструкции, оптимально соответствующие режимам эксплуатации. Таким образом, изучение влияния характера анизотропии свойств материалов на напряженно-деформированное состояние эксплуатируемых и вновь создаваемых конструкций составных сферических резервуаров (газгольдеры или шаровые хранилища) является актуальным.

Кроме того, аналитические решения позволяют установить и проиллюстрировать качественные эффекты, вызванные предельными переходами (геометрия, соотношение деформационных свойств и т.п.). В отличие от численных или численно-аналитических методов, применение которых не может гарантировать полноту проводимых исследований для выявления экстремальных и предельных случаев, аналитические решения не требуют больших объемов вычислений и при этом позволяют выявить характерные закономерности поведения полей напряжений и деформаций в элементах конструкций, закономерности их деформирования, а также пределы применимости используемых гипотез.

Несмотря на то, что многие современные конструкционные и функциональные композиционные материалы имеют ярко выраженную анизотропию 
деформационных и прочностных свойств, большинство авторов ограничивается лишь частным случаем, когда справедливо предположение об их изотропии, а количество работ, где учитываются другие типы упругой симметрии материалов, весьма ограничено. Так, например, решение задачи о равновесии упругой анизотропной полой сферы, приведенное в монографии С. Г. Лехницкого [1], было получено впервые в 1865 г. B. Saint-Venant [2]. Paвновесие анизотропных сфер рассматривается также в работе [3], а в статье [4] получено решение задачи о центрально-симметричной деформации полого шара из упругого радиально неоднородного трансверсально-изотропного материала, с помощью которого установлено наличие эффектов, вызванных неоднородностью, и проведено их качественное исследование. В работах [5-7] получены решения задач о равновесии упругих центрально-симметричных тел с закреплением на внешней или внутренней поверхности, находящихся в поле гравитационных сил под действием равномерных внутреннего или внешнего давлений соответственно. В настоящей работе для изучения влияния анизотропии материалов на характер распределения напряжений в поперечных сечениях составных сфер рассмотрен более простой случай, когда при решении краевой задачи отсутствует необходимость учета массовых сил.

\section{2. Аналитическое решение задачи Ламе для составной транс-} версально-изотропной сферы. Рассмотрим составное толстостенное центрально-симметричное тело, на которое действует равномерно распределенное внутреннее $p_{1}$ и внешнее $p_{2}$ давление. Будем считать, что тело состоит из двух полых толстостенных сфер с общим центром, в который поместим начало сферической ортогональной системы координат $\rho, \theta$ и $\varphi$. Материал каждой из сфер однородный, трансверсально-изотропный относительно любого радиус-вектора, проведенного из общего центра в данную точку. Будем предполагать, что составное тело ограничено сферическими поверхностями с радиусами $\rho_{1}$ и $\rho_{2}\left(\rho_{1}<\rho_{2}\right)$. Кроме того, в результате проведения технологических операций по изготовлению составные толстостенные сферические части тела образуют неразъемное соединение (достигается посадкой с натягом), объединены в единую механическую систему с известной поверхностью контакта между ними, находящейся на расстоянии $\rho_{c}$ от общего центра. Примем еще одну гипотезу: в процессе создания конструкции (в силу ее центральной симметрии) внешний радиус внутренней сферы уменьшился на величину радиального перемещения $u^{(1)}$ (имеет отрицательный по отношению к направлению радиальной координаты знак), а внутренний радиус внешней сферы увеличился на величину $u^{(2)}$. Обратим внимание на то, что алгебраическая сумма $u^{(1)}$ и $u^{(2)}$ равна величине натяга $\delta$. В дальнейшем все константы и функции, относящиеся к внутренней сфере, будем обозначать стоящими в круглых скобках верхним индексом (1), а к внешней - индексом (2) соответственно.

Поскольку составное сферическое тело обладает центральной симметрией и нагрузка, действующая на него, также центрально-симметрична, то отсутствуют сдвиги. Поэтому все точки будут перемещаться при нагружении только в радиальном направлении. Как следствие - напряжения, деформации и перемещения зависят только от одной переменной $\rho$. Тогда геометрические соотношения и уравнения равновесия для внутренней $(\alpha=1)$ и внешней 
$(\alpha=2)$ частей составной сферы записываются следующим образом:

$$
\begin{gathered}
\varepsilon_{\rho \rho}^{(\alpha)}=d u^{(\alpha)} / d \rho, \quad \varepsilon_{\varphi \varphi}^{(\alpha)}=\varepsilon_{\theta \theta}^{(\alpha)}=u^{(\alpha)} / \rho, \\
d \sigma_{\rho \rho}^{(\alpha)} / d \rho+2\left(\sigma_{\rho \rho}^{(\alpha)}-\sigma_{\varphi \varphi}^{(\alpha)}\right) / \rho=0
\end{gathered}
$$

а определяющие соотношения для трансверсально-изотропного тела в сферической системе координат

$$
\begin{aligned}
& \sigma_{\rho \rho}^{(\alpha)}=A_{11}^{(\alpha)} \varepsilon_{\rho \rho}^{(\alpha)}+A_{12}^{(\alpha)}\left(\varepsilon_{\varphi \varphi}^{(\alpha)}+\varepsilon_{\theta \theta}^{(\alpha)}\right), \\
& \sigma_{\varphi \varphi}^{(\alpha)}=A_{12}^{(\alpha)} \varepsilon_{\rho \rho}^{(\alpha)}+A_{22}^{(\alpha)} \varepsilon_{\varphi \varphi}^{(\alpha)}+A_{23}^{(\alpha)} \varepsilon_{\theta \theta}^{(\alpha)}, \\
& \sigma_{\theta \theta}^{(\alpha)}=A_{12}^{(\alpha)} \varepsilon_{\rho \rho}^{(\alpha)}+A_{23}^{(\alpha)} \varepsilon_{\varphi \varphi}^{(\alpha)}+A_{22}^{(\alpha)} \varepsilon_{\theta \theta}^{(\alpha)}
\end{aligned}
$$

содержат коэффициенты

$$
\begin{gathered}
A_{11}^{(\alpha)}=\tilde{E}^{(\alpha)}\left(1-\nu^{(\alpha)}\right) / m^{(\alpha)}, \quad A_{12}^{(\alpha)}=E^{(\alpha)} \tilde{\nu}^{(\alpha)} / m^{(\alpha)} \\
A_{23}^{(\alpha)}=E^{(\alpha)}\left(\nu^{(\alpha)}+s^{(\alpha)}\right) /\left(m^{(\alpha)}+\nu^{(\alpha)} m^{(\alpha)}\right), \quad s^{(\alpha)}=\tilde{\nu}^{(\alpha) 2} E^{(\alpha)} / \tilde{E}^{(\alpha)}, \\
A_{22}^{(\alpha)}=E^{(\alpha)}\left(1-s^{(\alpha)}\right) /\left(m^{(\alpha)}+\nu^{(\alpha)} m^{(\alpha)}\right), \quad m^{(\alpha)}=1-\nu^{(\alpha)}-2 s^{(\alpha)}
\end{gathered}
$$

вычисляемые при помощи упругих постоянных. Здесь $\tilde{E}^{(\alpha)}$ и $E^{(\alpha)}$ - модули Юнга для растяжения вдоль радиус-вектора $\rho$ и в перпендикулярном к нему направлении; $\tilde{\nu}^{(\alpha)}$ - коэффициент Пуассона, характеризующий поперечную деформацию при растяжении в направлении $\rho ; \nu^{(\alpha)}-$ коэффициент Пуассона, характеризующий сокращение в плоскости, нормальной к радиус-вектору $\rho$ при растяжении в той же плоскости.

Последовательная подстановка геометрических соотношений (1) в определяющие (3), а полученного результата - в уравнения равновесия (2) позволяет записать обыкновенные дифференциальные уравнения второго порядка:

$$
2\left(A_{12}^{(\alpha)}-A_{22}^{(\alpha)}-A_{23}^{(\alpha)}\right) u^{(\alpha)}+2 \rho A_{11}^{(\alpha)} d u^{(\alpha)} / d \rho+\rho^{2} A_{11}^{(\alpha)} d^{2} u^{(\alpha)} / d \rho^{2}=0,
$$

общие решения которых выглядят следующим образом [1]:

$$
u^{(\alpha)}=\rho^{-1 / 2-k^{(\alpha)}}\left(C_{1}^{(\alpha)}+\rho^{2 k^{(\alpha)}} C_{2}^{(\alpha)}\right) .
$$

Здесь $k^{(\alpha)}=\sqrt{1 / 4+2\left(A_{22}^{(\alpha)}+A_{23}^{(\alpha)}-A_{12}^{(\alpha)}\right)} / A_{11}^{(\alpha)}-$ показатель анизотропии для центрально-симметричного тела, находящегося под действием центральносимметричной нагрузки (введенный С. Г. Лехницким [1]), который для изотропной среды принимает значение $3 / 2$.

Определение перемещений и напряжений в составном центрально-симметричном теле (в силу линейной упругости его составных частей) осуществляется в результате суперпозиции решений следующих задач: составная сфера находится в равновесии под действием равномерно распределенных внутреннего и внешнего давлений (первая задача) и две отдельно рассматриваемые части составной сферы, находящиеся под действием технологических напряжений (на внутренней или внешней поверхности радиуса $\rho_{c}$ ) при отсутствии других внешних силовых воздействий (вторая задача). 
В процессе создания составных конструкций возникают собственные технологические напряжения, определение закона распределения которых в поперечных сечениях представляет самостоятельную задачу, выходящую за рамки настоящего исследования. Будем предполагать, что эти напряжения известны, имеют единственную, отличную от нуля, радиальную составляющую и равномерно распределены только на поверхности контакта радиуса $\rho_{c}$. Будем также считать, что остальные поверхности составных частей анизотропной сферы свободны от собственных напряжений. Сделаем еще одно предположение: радиальное напряжение (давление) натяга на известной поверхности контакта, вызванное технологическими операциями процесса изготовления конструкции, обусловлено только разницей в параметрах геометрии внутренней и внешней частей.

Итак, сумма перемещений на поверхности контакта равна величине натяга:

$$
\left.u^{(2)}\right|_{\rho=c}-\left.u^{(1)}\right|_{\rho=c}=\delta .
$$

Рассмотрим решение второй задачи для внутренней и внешней части составного центрально-симметричного тела. Для этой задачи граничные условия

$$
\left.\sigma_{\rho \rho}^{(1)}\right|_{\rho=\rho_{c}}=\left.\sigma_{\rho \rho}^{(2)}\right|_{\rho=\rho_{c}}=-p_{\delta},\left.\quad \sigma_{\rho \rho}^{(1)}\right|_{\rho=\rho_{1}}=\left.\sigma_{\rho \rho}^{(2)}\right|_{\rho=\rho_{2}}=0
$$

позволяют найти константы интегрирования, подстановка которых в уравнения (4) и условие (5) позволяет определить контактное давление натяга:

$$
p_{\delta}=\frac{\delta}{2 \rho_{c}}\left(b_{+}^{(1)} b_{-}^{(1)} b_{+}^{(2)} b_{-}^{(2)} c_{1} c_{2}\right) Z_{2},
$$

где

$$
\begin{aligned}
& b_{+}^{(\alpha)}=4 A_{12}^{(\alpha)}-A_{11}^{(\alpha)}\left(1+2 k^{(\alpha)}\right), \quad b_{-}^{(\alpha)}=4 A_{12}^{(\alpha)}-A_{11}^{(\alpha)}\left(1-2 k^{(\alpha)}\right), \\
& c_{\alpha}=\rho_{c}^{2 k^{(\alpha)}}-\rho_{\alpha}^{2 k^{(\alpha)}}, \quad g_{\alpha}=b_{+}^{(\alpha)} \rho_{c}^{2 k^{(\alpha)}}-b_{-}^{(\alpha)} \rho_{\alpha}^{2 k^{(\alpha)}}, \quad q_{\alpha}=c_{\alpha} b_{+}^{(\alpha)} b_{-}^{(\alpha)}, \\
& Z_{\alpha}=g_{\beta} q_{\alpha}-g_{\alpha} q_{\beta}, \quad \beta= \begin{cases}1, & \alpha=2, \\
2, & \alpha=1 .\end{cases}
\end{aligned}
$$

Перемещения, возникающие в составной сфере, находящейся в равновесии под действием равномерных внутреннего и внешнего давлений (первая задача), определяются уравнениями (4), а константы интегрирования $C_{1}^{(\alpha)}$ и $C_{2}^{(\alpha)}$, входящие в него, находятся из граничных условий

$$
\begin{gathered}
\left.\sigma_{\rho \rho}^{(1)}\right|_{\rho=\rho_{1}}=-p_{1},\left.\quad \sigma_{\rho \rho}^{(2)}\right|_{\rho=\rho_{2}}=-p_{2}, \\
\left.\sigma_{\rho \rho}^{(1)}\right|_{\rho=\rho_{c}}=\left.\sigma_{\rho \rho}^{(2)}\right|_{\rho=\rho_{c}},\left.\quad u^{(1)}\right|_{\rho=\rho_{c}}=\left.u^{(2)}\right|_{\rho=\rho_{c}}
\end{gathered}
$$

и записываются следующим образом:

$$
\begin{aligned}
Z_{\alpha} C_{1}^{(\alpha)}=2 \rho_{c}^{k^{(\alpha)}} \rho_{\alpha}^{k^{(\alpha)}}\left[p _ { \alpha } \rho _ { c } ^ { k ^ { ( \alpha ) } } \rho _ { \alpha } ^ { 3 / 2 } \left(q_{\beta}-\right.\right. & \left.g_{\beta} b_{-}^{(\alpha)}\right)- \\
& \left.-4 p_{\beta} A_{11}^{(\beta)} k^{(\beta)} b_{-}^{(\alpha)} \rho_{c}^{k^{(\beta)}} \rho_{\alpha}^{k^{(\alpha)}} \rho_{\beta}^{3 / 2+k^{(\beta)}}\right]
\end{aligned}
$$




$$
Z_{\alpha} C_{2}^{(\alpha)}=2\left[4 p_{\beta} A_{11}^{(\beta)} b_{+}^{(\alpha)} k^{(\beta)} \rho_{c}^{k^{(\alpha)}+k^{(\beta)}} \rho_{\beta}^{3 / 2+k^{(\beta)}}-p_{\alpha} \rho_{\alpha}^{3 / 2+k^{(\alpha)}}\left(q_{\beta}-g_{\beta} b_{+}^{(\alpha)}\right)\right]
$$

По известным после определения постоянных $C_{1}^{(\alpha)}$ и $C_{2}^{(\alpha)}$ перемещениям из уравнений (1) могут быть определены деформации:

$$
\begin{aligned}
& 2 \varepsilon_{\rho \rho}^{(\alpha)}=-\rho^{-3 / 2-k^{(\alpha)}}\left[C_{1}^{(\alpha)}\left(1+2 k^{(\alpha)}\right)+C_{2}^{(\alpha)}\left(1-2 k^{(\alpha)}\right) \rho^{2 k^{(\alpha)}}\right], \\
& \varepsilon_{\theta \theta}^{(\alpha)}=\varepsilon_{\varphi \varphi}^{(\alpha)}=\rho^{-3 / 2-k^{(\alpha)}}\left(C_{1}^{(\alpha)}+C_{2}^{(\alpha)} \rho^{2 k^{(\alpha)}}\right),
\end{aligned}
$$

а из уравнений (3) - напряжения:

$$
\begin{aligned}
& 2 \sigma_{\rho \rho}^{(\alpha)}=\rho^{-3 / 2-k^{(\alpha)}}\left(C_{1}^{(\alpha)} b_{+}^{(\alpha)}+C_{2}^{(\alpha)} b_{-}^{(\alpha)} \rho^{2 k^{(\alpha)}}\right), \\
& 2 \sigma_{\theta \theta}^{(\alpha)}=2 \sigma_{\varphi \varphi}^{(\alpha)}=\rho^{-3 / 2-k^{(\alpha)}}\left(C_{1}^{(\alpha)} B_{+}^{(\alpha)}+C_{2}^{(\alpha)} B_{-}^{(\alpha)} \rho^{2 k^{(\alpha)}}\right),
\end{aligned}
$$

где

$$
\begin{gathered}
B_{+}^{(\alpha)}=2 Q^{(\alpha)}-A_{12}^{(\alpha)}\left(1+2 k^{(\alpha)}\right), \quad B_{-}^{(\alpha)}=2 Q^{(\alpha)}-A_{12}^{(\alpha)}\left(1-2 k^{(\alpha)}\right), \\
Q^{(\alpha)}=A_{22}^{(\alpha)}+A_{23}^{(\alpha)} .
\end{gathered}
$$

В частном случае для изотропной составной сферы проведем замену

$$
\tilde{E}^{(\alpha)}=E^{(\alpha)}, \quad \tilde{\nu}^{(\alpha)}=\nu^{(\alpha)}
$$

значительно упростим коэффициент $A_{11}^{(\alpha)}$ :

$$
A_{11}^{(\alpha)}=E^{(\alpha)}\left(1-\nu^{(\alpha)}\right) /\left[\left(1+\nu^{(\alpha)}\right)\left(1-2 \nu^{(\alpha)}\right)\right]
$$

и запишем выражения для перемещений, деформаций и напряжений:

$$
\begin{gathered}
\rho^{2} u^{(\alpha)}=C_{1}^{(\alpha)}+C_{2}^{(\alpha)} \rho^{3}, \\
\varepsilon_{\rho \rho}^{(\alpha)}=C_{2}^{(\alpha)}-2 C_{1}^{(\alpha)} / \rho^{3}, \quad \varepsilon_{\theta \theta}^{(\alpha)}=\varepsilon_{\varphi \varphi}^{(\alpha)}=C_{1}^{(\alpha)} / \rho^{3}+C_{2}^{(\alpha)}, \\
\sigma_{\rho \rho}^{(\alpha)}=\left(C_{1}^{(\alpha)} b_{+}^{(\alpha)}+C_{2}^{(\alpha)} b_{-}^{(\alpha)} \rho^{3}\right) /\left(2 \rho^{3}\right), \\
\sigma_{\theta \theta}^{(\alpha)}=\sigma_{\varphi \varphi}^{(\alpha)}=C_{2}^{(\alpha)} b_{-}^{(\alpha)} / 2-C_{1}^{(\alpha)} b_{+}^{(\alpha)} /\left(4 \rho^{3}\right), \\
Z_{\alpha} C_{1}^{(\alpha)}=2\left[p_{\alpha}\left(c_{\beta} b_{+}^{(\beta)} b_{-}^{(\beta)}-g_{\beta} b_{-}^{(\alpha)}\right)-6 p_{\beta} A_{11}^{(\beta)} b_{-}^{(\alpha)} \rho_{\beta}^{3}\right] \rho_{c}^{3} \rho_{\alpha}^{3}, \\
Z_{\alpha} C_{2}^{(\alpha)}=2\left[6 p_{\beta} A_{11}^{(\beta)} b_{+}^{(\alpha)} \rho_{c}^{3} \rho_{\beta}^{3}-p_{\alpha} \rho_{\alpha}^{3}\left(c_{\beta} b_{+}^{(\beta)} b_{-}^{(\beta)}-b_{+}^{(\alpha)} g_{\beta}\right)\right], \\
c_{\alpha}=\rho_{c}^{3}-\rho_{\alpha}^{3}, \quad g_{\alpha}=b_{+}^{(\alpha)} \rho_{c}^{3}-b_{-}^{(\alpha)} \rho_{\alpha}^{3}, \\
b_{+}^{(\alpha)}=-4 E^{(\alpha)} /\left(1+\nu^{(\alpha)}\right), \quad b_{-}^{(\alpha)}=2 E^{(\alpha)} /\left(1-2 \nu^{(\alpha)}\right) .
\end{gathered}
$$

Если замену упругих постоянных (8) провести в $(4),(6)$ и (7) только в выражениях, записанных для одной из частей составной сферы, то получим соотношения еще для двух частных случаев: решение задачи о равновесии составной центрально-симметричной конструкции, состоящей из изотропной внутренней или внешней и трансверсально-изотропной внешней или внутренней частей соответственно. 
3. Влияние анизотропии и технологического давления на напряженное состояние составных сферических сосудов. Полученные выражения позволяют найти распределение напряжений в поперечных сечениях составных сферических сосудов и определить закономерности взаимного влияния внутренней и внешней частей, обусловленные анизотропией материалов и величин давления на поверхности контакта, а также провести многокритериальную оценку начальной прочности.

На основе введенных в работе [8] величин

$$
\begin{gathered}
2 J_{\sigma}^{\mathrm{I}}=\sigma_{\varphi \varphi}+\sigma_{\theta \theta}, \\
J_{\sigma}^{\mathrm{III}}=\sqrt{\left(\sigma_{\varphi \varphi}-\sigma_{\theta \theta}\right)^{2}+4 \sigma_{\varphi \theta}^{2}}, \quad J_{\sigma}^{\mathrm{IV}}=\sqrt{\sigma_{\varphi \rho}^{2}+\sigma_{\theta \rho}^{2}}
\end{gathered}
$$

инвариантных относительно ортогональных преобразований, допустимых над трансверсально-изотропным телом, авторами [9] были сконструированы критериальные условия, позволяющие описать различные механизмы исчерпания несущей способности.

Поскольку составные сферические сосуды давления и характер нагрузки, действующей на них, центрально-симметричны, то отсуствуют сдвиговые деформации и касательные напряжения. Поэтому $J_{\sigma}^{\mathrm{III}}=J_{\sigma}^{\mathrm{IV}}=0$.

На рис. 1 и рис. 2 представлены распределения ненулевых инвариантов тензора напряжений $J_{\sigma}^{(\bullet)}$ в поперечных сечениях составных сферических сосудов давления, находящихся под действием внутреннего $p_{1}=1$ МПа и внешнего $p_{2}=5$ МПа равномерных давлений, вдоль обезразмеренной радиальной координаты $\tilde{\rho}=\left(\rho-\rho_{1}\right) /\left(\rho_{2}-\rho_{1}\right)$ в зависимости от показателя анизотропии материала внутренней и внешней части соответственно. Эти распределения получены на основе решения первой задачи. В расчетах полагалось, что $\rho_{1}=3 \mathrm{~m}, \rho_{c}=4.7 \mathrm{M}, \rho_{2}=6.0 \mathrm{M}$. А деформационные характеристики, соответствующие различным значениям показателя $k^{(\alpha)}$ (см. таблицу), выбирались с учетом положительности собственных значений оператора упругости для трансверсально-изотропного тела [10]:

$$
\begin{gathered}
A_{22}^{(\alpha)}-A_{23}^{(\alpha)}>0 \\
A_{11}^{(\alpha)}+A_{22}^{(\alpha)}+A_{23}^{(\alpha)} \pm \sqrt{8 A_{12}^{(\alpha) 2}+\left(A_{22}^{(\alpha)}+A_{23}^{(\alpha)}-A_{11}^{(\alpha)}\right)^{2}}>0
\end{gathered}
$$

Представленные на рис. 1 и рис. 2 результаты отражают влияние анизотропии материалов внешней и внутренней частей составного сосуда соответственно. При увеличении показателя анизотропии $k^{(1)}$ происходит увеличение абсолютной величины первого $J_{\sigma}^{\mathrm{I}}$ и второго $J_{\sigma}^{\mathrm{II}}$ инвариантов тензора

Деформационные характеристики материалов [Materials constants]

\begin{tabular}{c||c|c|c|c}
\hline$k^{(\alpha)}$ & $E^{(\alpha)}, \mathrm{GPa}$ & $\tilde{E}^{(\alpha)}, \mathrm{GPa}$ & $\nu^{(\alpha)}$ & $\tilde{\nu}^{(\alpha)}$ \\
\hline 2.198 & 55.0 & 23.0 & 0.29 & 0.32 \\
2.349 & 55.0 & 20.0 & 0.29 & 0.32 \\
1.500 & 45.0 & 45.0 & 0.30 & 0.30 \\
1.060 & 23.0 & 55.0 & 0.32 & 0.29 \\
1.005 & 20.0 & 55.0 & 0.32 & 0.29 \\
\hline
\end{tabular}




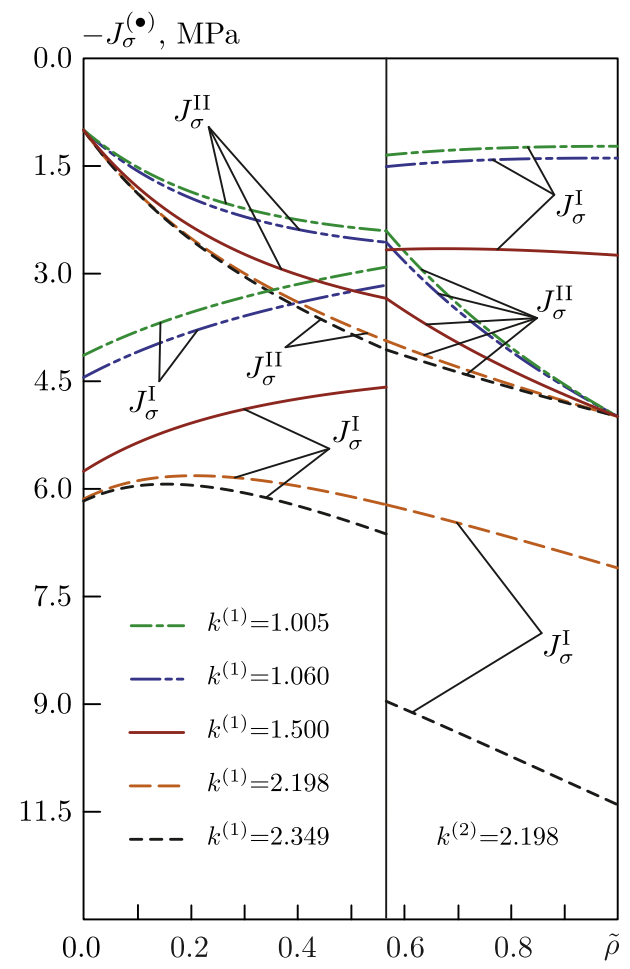

Рис. 1. Распределение инвариантов тензора напряжений в поперечных сечениях составных сферических сосудов в зависимости от показателя анизотропии материала внутренней части

[Figure 1. Distribution of stress tensor invariants in cross sections of combined pressure vessels depending on the anisotropy index of the material of the inner part]

напряжений и во внутренней, и во внешней части составной конструкции. Вместе с тем увеличение показателя анизотропии $k^{(2)}$, напротив, приводит к уменьшению по абсолютной величине $J_{\sigma}^{\mathrm{I}}$ и $J_{\sigma}^{\mathrm{II}}$. Изотропному материалу соответствует $k^{(\alpha)}=1.5$.

Оценка начальной прочности позволяет сделать вывод, что разрушение от растяжения или сжатия в радиальном направлении может начаться в точках внешней поверхности, на которую действует большее давление, а, соответственно, второй инвариант тензора напряжений $J_{\sigma}^{\mathrm{II}}$ имеет большее (по абсолютной величине) значение, либо на поверхности контакта, если показатель анизотропии материала внутренней части составной сферы будет больше, чем у внешней. С точки зрения возможности начала разрушения от растяжения или сжатия в окружном направлении наиболее опасными являются точки внешней, или контактной, поверхности при условии, что $k^{(1)} \geqslant k^{(2)}$, а при $k^{(1)}<k^{(2)}$ - точки внутренней поверхности составной центрально-симметричной конструкции.

Обнаруженные закономерности влияния анизотропии материалов внутренней и внешней частей сферических сосудов давления свидетельствуют о возможности «управления» величинами и характером распределения напряжений при проектировании составных элементов конструкций, оптимально соответствующих заданным режимам эксплуатации. Это «управление», на- 


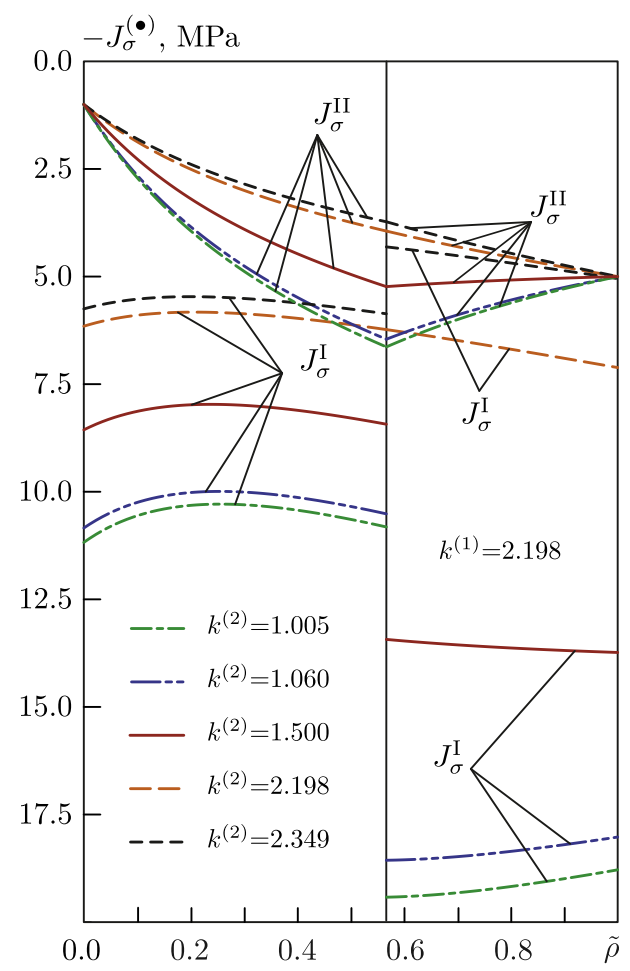

Рис. 2. Распределение инвариантов тензора напряжений в поперечных сечениях составных сферических сосудов в зависимости от показателя анизотропии материала внешней части [Figure 2. Distribution of stress tensor invariants in cross sections of combined pressure vessels depending on the anisotropy index of the material of the outer part]

пример, может быть реализовано только изменением схемы армирования анизотропного материала при сохранении свойств отдельных элементов структуры.

Обратим внимание на то, что в частном случае, когда составные части сосуда давления изготовлены из одного и того же материала, составную конструкцию можно рассматривать как однородную толстостенную сферу. Тогда на поверхности контакта, определяемой $\rho_{c}$, будут выполняться условия непрерывности для всех компонент тензора напряжений, а, следовательно, полученные соотношения будут соответствовать решению задачи Ламе для однородной толстостенной трансверсально-изотропной сферы, находящейся под действием распределенных внешнего и внутреннего давлений [1].

На рис. 3 показано влияние контактного давления натяга $p_{\delta}$, равномерно распределенного на поверхности контакта, на зависимости ненулевых инвариантов тензора напряжений от координаты $\tilde{\rho}$ в поперечных сечениях составных сосудов давления, изготовленных из трансверсально-изотропных материалов и находящихся под действием внутреннего $p_{1}=1 \mathrm{MПа} \mathrm{и} \mathrm{внешнего}$ $p_{2}=5$ МПа равномерных давлений. Это оказалось возможным благодаря использованию суперпозиции решений первой и второй задач. Геометрические параметры и упругие постоянные материалов частей центрально-симметричной конструкции полагались следующими: $\rho_{1}=3 \mathrm{M}, \rho_{c}=4.7 \mathrm{м} \mathrm{и} \rho_{2}=6.0 \mathrm{M}$; $E^{(\alpha)}=55$ ГПа, $\tilde{E}^{(\alpha)}=23$ ГПа, $\nu^{(\alpha)}=0.29$ и $\tilde{\nu}^{(\alpha)}=0.32$. 


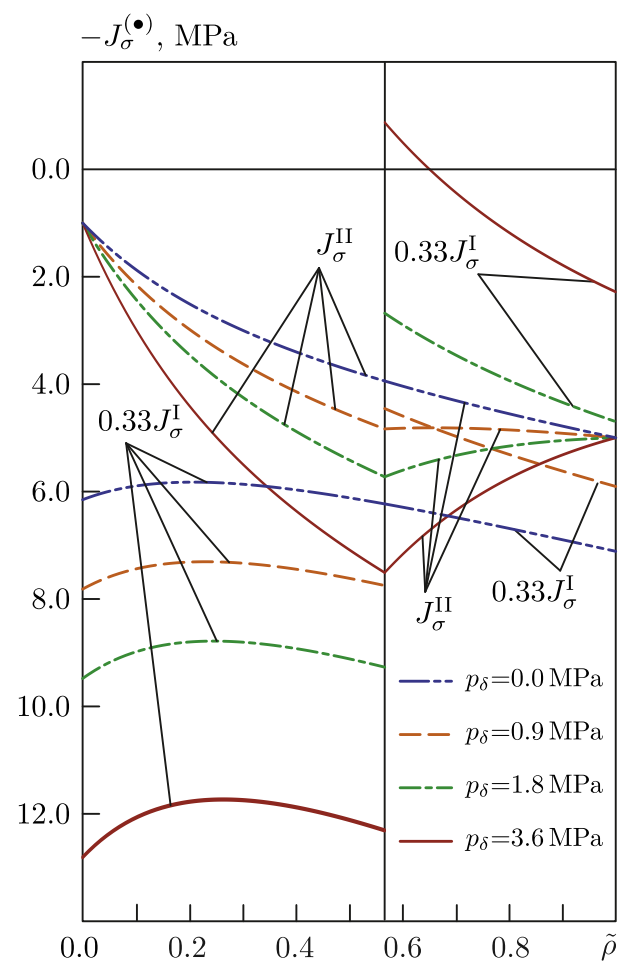

Рис. 3. Распределение инвариантов тензора напряжений в поперечных сечениях составных сферических сосудов в зависимости от величин технологического давления

[Figure 3. Distribution of stress tensor invariants in cross sections of combined pressure vessels depending on the contact process pressure values]

С увеличением технологического давления натяга $p_{\delta}$ возрастает $J_{\sigma}^{\mathrm{II}}$ и увеличивается скачок $J_{\sigma}^{\mathrm{I}}$ на поверхности контакта, при этом характер распределения напряжений в поперечных сечениях составных сферических сосудов давления становится более нелинейным. Это приводит к тому, что наиболее опасными с точки зрения возможности начала разрушения становятся (по сравнению с $p_{\delta}=0.0 \mathrm{MПа)} \mathrm{другие} \mathrm{точки} \mathrm{в} \mathrm{конструкции.} \mathrm{Так,} \mathrm{например,} \mathrm{при}$ $p_{\delta}>0.9$ МПа разрушение по механизму сжатия в окружном направлении может быть инициировано в точках, принадлежащих внутренней поверхности составной конструкции. Последнее имеет место, несмотря но то, что внешнее давление по абсолютной величине выше внутреннего и превосходит давление натяга. Точки составного сосуда давления, находящиеся на поверхности контакта, становятся наиболее опасными с точки зрения возможности начала разрушения по механизму сжатия в радиальном направлении.

4. Заключение. Полученное точное аналитическое решение задачи Ламе для составных трансверсально-изотропных сфер с общим центром могут быть использованы как для определения полей перемещений, деформаций и напряжений в сферических резервуарах (газгольдеры или шаровые хранилища для находящихся под избыточным давлением сжиженных газов, высокоагрессивных сред и легковоспламеняющихся жидкостей) [11], так и для «управления» напряженным состоянием составных сферических конструкций при их проектировании с целью увеличения эффективности. Кроме того, 
полученные результаты будут полезны для тестирования алгоритмов численного решения задач для сферических конструкций, изготовленных из анизотропных материалов, и при отработке методик экспериментов для центрально-симметричных тел.

Конкурирующие интересы. Заявляем, что в отношении авторства и публикации этой статьи конфликта интересов не имеем.

Авторский вклад и ответственность. А.В. Зайцев- идея исследования, получение аналитических решений и их анализ, аналитический обзор, черновик и чистовик рукописи. Ю.В. Соколкин - идея исследования, формулировка целей и задач исследования, анализ аналитических решений, консультирование, работа с черновиком рукописи. А.А. Фукалов - получение аналитических решений и их анализ, визуализация и верификация результатов, черновик и чистовик рукописи. Авторы несут полную ответственность за предоставление окончательной рукописи в печать. Окончательная версия рукописи была одобрена всеми авторами.

Финансирование. Работа выполнена в рамках государственного задания Минобрнауки РФ FSNM-2020-0027 на выполнение фундаментальных научных исследований на 2020 г. и плановый период 2021 и 2022 гг. и при поддержке Российского фонда фундаментальных исследований (грант РФФИ-Урал № 19-41-590026_а).

\section{Библиографический список}

1. Лехницкий С. Г. Теория упругости анизотропного тела. М.: Наука, 1977. 416 с.

2. Saint-Venant B. Mémoire sur les divers genres d'homogénéité semi-polaire ou cylindrique et sur les homogénéités polaires ou sphéri-coniques et sphériques // J. Math. Pures Appl., 1865. vol. 10. pp. 297-349.

3. Шармазанашвили А. Х. Расчет анизотропных толстостенных сферических оболочек // Вестн. инж. и техников, 1938. № 7. С. 35-37.

4. Колчин Г. Б., Ковалов Е. К. Центрально-симметричная деформация упругого радиально-неоднородного трансверсально-изотропного полого шара // Изв. РАН. MTT, 1995. № 6. C. 42-47.

5. Зайцев А. В., Фукалов А. А. Упругое равновесие тяжелой трансверсально-изотропной толстостенной сферы с жёстко закреплённой внутренней поверхностью // Becmн. Caм. гос. техн. ун-та. Сер. Физ.-мат. науки, 2010. №5(21). С. 85-95. https://doi.org/ $10.14498 /$ vsgtu818.

6. Зайцев А. В., Соколкин Ю. В., Фукалов А. А. Механизмы начального разрушения железобетонной крепи сферической горной выработки в массиве осадочных пород // Вестник Пермского национального исследовательского политехнического университета. Механика, 2013. №4. C. 59-74. https://doi.org/10.15593/perm.mech/2013.4. 59-74.

7. Zaitsev A. V., Fukalov A. A., Sokolkin Y. V. Initial strength analysis of anisotropic concrete supports for spherical mine workings in a sedimentary rock mass / Physical and Mathematical Modeling of Earth and Environment Processes. Cham: Springer, 2019. pp. 463-471. https://doi.org/10.1007/978-3-030-11533-3_46.

8. Победря Б. Е. Механика композичионных материалов. М.: МГУ, 1984. 336 с.

9. Вильдеман В. Э., Соколкин Ю. В., Ташкинов А. А. Механика неупругого деформирования и разрушения композиционных материалов. М.: Наука, 1997. 288 с.

10. Mityushov E. A., Berestova S. A., Odintsova N. Yu. Effective elastic properties of textured cubic polycrystals // Texture, Stress, and Microstructure, 2002. vol.35, no. 2. pp. 99-111. https://doi.org/10.1080/0730330021000000227.

11. Бобрицкий Н. В., Юфин В. А. Основы нефтяной и газовой промышленности. М.: Недра, 1988. 200 c. 


\title{
MSC: 74B05
}

\section{Solution of the Lamé problem for combined transversely isotropic spheres with a general center}

\author{
(C) A. V. Zaitsev, Yu. V. Sokolkin, A. A. Fukalov
}

Perm State National Research Polytechnical University,

29, Komsomolskiy pr., 614990, Perm, Russian Federation.

\begin{abstract}
The paper deals with obtaining an exact analytical solution to the Lamé problem on the equilibrium state of a combined body consisting of two tightly fitted transversely isotropic spheres with a common center. The body is influenced by uniformly distributed external and internal pressures. The process pressure on the contact surface is determined assuming that it is a consequence of the difference in the geometry of the individual parts of the combined sphere only. We analyzed the laws of the influence of the materials' anisotropy (the material constants satisfy the relations in the form of inequalities that ensure the positivity of the eigenvalues of the elasticity operator) and the values of the contact process pressure on the stress distribution in the cross sections of pressure vessels. The influence assessment of the materials' anisotropy shows an opportunity to control the values and nature of the stress distribution in the combined structures that are optimal for the specified operating conditions. The obtained results indicate that a change in the anisotropy index, i.e. an increase in its values in the inner or outer parts of the spheres leads to an increase or decrease in the absolute values of stresses, respectively. This increase or decrease in the anisotropy indices can be realized at the stage of structures' design due to a change in the reinforcement scheme while maintaining the properties of the individual structural elements. Based on a multicriteria approach, the initial strength of combined pressure vessels was estimated using the mechanisms of tension or compression in the radial and hoop directions. It was found that an increase in the pressure on the contact surface can lead to the material domains that do not resist compression in the hoop direction. These domains
\end{abstract}

\section{Research Article}

( ) (i) The content is published under the terms of the Creative Commons Attribution 4.0 International License (http://creativecommons.org/licenses/by/4.0/)

Please cite this article in press as:

Z aits ev A. V., Sokolk in Yu. V., Fukalov A. A. Solution of the Lamé problem for combined transversely isotropic spheres with a general center, Vestn. Samar. Gos. Tekhn. Univ., Ser. Fiz.Mat. Nauki [J. Samara State Tech. Univ., Ser. Phys. Math. Sci.], 2021, vol. 25, no. 1, pp. 83-96. https://doi.org/10.14498/vsgtu1830 (In Russian).

\section{Authors' Details:}

Alexey V. Zaitsev (1) https://orcid.org/0000-0003-0578-7917

Cand. Phys. \& Math. Sci.; Associate Professor; Dept. of Mechanics of Composite Material and Structures; e-mail: a-zaitsev@mail.ru

Yuriy V. Sokolkin (10 https://orcid.org/0000-0003-3255-1360

Dr. Phys. \& Math. Sci.; Professor; Dept. of Mechanics of Composite Material and Structures

Anton A. Fukalov (1) https://orcid.org/0000-0003-3009-7379

Senior Lecturer; Dept. of Mechanics of Composite Material and Structures;

e-mail: mr_aa@mail.ru 
are located in the vicinity of the internal surface of the vessel, on which a uniformly distributed pressure acts, which is less in the absolute value than the external pressure. It was found that the points of the combined vessel located on the contact surface become most dangerous from the point of beginning the damage by the compression in the radial direction.

Keywords: analytical solution, Lamé problem, combined transversely isotropic sphere, anisotropy, multi-criteria evaluation of initial strength, combined spherical vessels, contact pressure.

Received: $5^{\text {th }}$ October, $2020 /$ Revised: $18^{\text {th }}$ February, $2021 /$

Accepted: $10^{\text {th }}$ March, $2021 /$ First online: $30^{\text {th }}$ March, 2021

Competing interests. We declare that we have no conflicts of interest in the authorship and publication of this article.

Authors' contributions and responsibilities. A.V. Zaitsev: Idea of study; Obtaining analytical solutions and their analysis; Literature review; Writing — original draft and review \& editing. Yu.V. Sokolkin: Idea of study; Formulation of research goals and aims; Consulting; Writing - original draft. A. A. Fukalov: Obtaining analytical solutions and their analysis; Visualization and verification of results; Writing — original draft and review \& editing. The authors are absolutely responsible for submitting the final manuscript in print. Each author has approved the final version of manuscript.

Funding. The study is supported by the Russian Ministry of Science and Education (State Assignment FSNM-2020-0027 for Basic Researches in 2020-2022) and by the Russian Foundation for Basic Research (project no. 19-41-590026_a).

\section{References}

1. Lekhnitskii S. G. Theory of Elasticity of an Anisotropic Body. Moscow, Mir Publ., 1981, 430 pp.

2. Saint-Venant B. Mémoire sur les divers genres d'homogénéité semi-polaire ou cylindrique et sur les homogénéités polaires ou sphéri-coniques et sphériques, J. Math. Pures Appl., 1865, vol. 10, pp. 297-349.

3 .

4. Kolchin G. B., Kovalov E. K. Centrally symmetric deformation of an elastic radially inhomogeneous transversely isotropic hollow sphere, Izv. Ross. Akad. Nauk. Mekh. Tverd. Tela, 1995, no. 6, pp. 42-47 (In Russian).

5. Zaitsev A. V., Fukalov A. A. Elastic equilibrium state of thick-walled heavy transverselyisotropic spheres fixed on the interior surface, Vestn. Samar. Gos. Tekhn. Univ., Ser. Fiz.Mat. Nauki [J. Samara State Tech. Univ., Ser. Phys. Math. Sci.], 2010, no. 5(21), pp. 85-95 (In Russian). https://doi.org/10.14498/vsgtu818.

6. Zaytsev A. V., Sokolkin Y. V., Fukalov A. A. Initial damage mechanisms of reinforced concrete monolithic supports for spherical mine workings located in sedimentary rock mass, PNRPU Mechanics Bulletin, 2013, no.4, pp. 54-74 (In Russian). https://doi.org/10. 15593/perm. mech/2013.4.59-74.

7. Zaitsev A. V., Fukalov A. A., Sokolkin Y. V. Initial strength analysis of anisotropic concrete supports for spherical mine workings in a sedimentary rock mass, In: Physical and Mathematical Modeling of Earth and Environment Processes. Cham, Springer, 2019, pp. 463-471. https://doi.org/10.1007/978-3-030-11533-3_46.

8. Pobedrya B. E. Mekhanika kompozitsionnykh materialov [Mechanics of Composite Materials]. Moscow, Moscow State Univ., 1984, 336 pp. (In Russian) 
9. Vildeman V. E., Sokolkin Yu. V., Tashkinov A. A. Mekhanika neuprugogo deformirovaniia $i$ razrusheniia kompozitsionnykh materialov [Mechanics of Inelastic Deformation and Fracture of Composite Materials]. Moscow, Nauka, 1997, 288 pp. (In Russian)

10. Mityushov E. A., Berestova S. A., Odintsova N. Yu. Effective elastic properties of textured cubic polycrystals, Texture, Stress, and Microstructure, 2002, vol.35, no.2, pp. 99-111. https://doi.org/10.1080/0730330021000000227.

11. Bobritskii N. V., Yufin V. A. Osnovy neftianoi i gazovoi promyshlennosti [Fundamentals of Oil and Gas Industry]. Moscow, Nedra, 1988, 200 pp. (In Russian) 